AED treatment of PS and rolandic epilepsy (RE). A clinical practice survey in the UK among 590 pediatricians who treat epilepsy found that $40 \%$ of 132 respondents reported non-treatment of PS and RE because of low frequency of seizures and parent/child preferences. They estimated 233 new cases of PS and 751 new RE cases, annually. Carbamazepine is the preferred older, and levetiracetam the preferred newer AED in randomized controlled trials [4].

\title{
References.
}

1. Berg AT, et al. Epilepsia. 2010 Apr;51(4):676-85.

2. Moseley B, et al. Epilepsy Behav. 2013 Mar;26(3):375-85.

3. Millichap JG, Lombroso CT, Lennox WG. Pediatrics. 1955 Jun;15(6):705-14.

4. Mellish LC, et al. Arch Dis Child. 2014 Sep 8. [Epub ahead of print].

\section{KCNB1 MUTATIONS IN EPILEPTIC ENCEPHALOPATHY}

Researchers at Scripps Research Institute, San Diego, and other centers in California; Johns Hopkins, Baltimore; Vanderbilt University, TN; and Northwestern University Feinberg School of Medicine, Chicago, IL; searched for de novo mutations in a family with a sporadic case of epileptic encephalopathy. The cause was determined using whole exome sequencing (WES) and whole genome sequencing (WGS). A de novo missense mutation in KCNB1 was identified that encodes the K2.1 voltage-gated potassium channel. Subsequently, 2 additional patients were identified with epileptic encephalopathy and de novo KCNB1 missense mutations that cause a similar pattern of K2.1 dysfunction. Clinical WES may be useful for diagnosis of epileptic encephalopathies of unknown etiology. (Torkamani A, Bersell K, et al. De novo KCNB1 mutations in epileptic encephalopathy. Ann Neurol 2014 Oct;76(4):529-40).

COMMENTARY. Researchers at University of Arizona, Tucson, previously explored the utility of WES and identified causal de novo variants in genes of 7 of 10 children with sporadic epilepsy, refractory seizures, developmental delay, or epileptic encephalopathy. These probands all presented with seizures within the first 6 months of life, and 6 have intractable seizures. The genes affected included SCN1A, CDKL5, EEF1A2, and KCNH5 [1]. The present finding of de novo KCNB1 mutations as a cause of K2.1 dysfunction expands the locus heterogeneity associated with epileptic encephalopathies [2].

\section{References.}

1. Veeramah KR, et al. Epilepsia. 2013 Jul;54(7):1270-81.

2. Torkamani A, et al. Ann Neurol. 2014 Oct;76(4):529-40.

\section{TRANSITION CARE TO ADULT EPILEPSY CENTERS}

Epileptologists at University of Toronto and University of Saskatchewan, Canada, evaluated the complexity of epilepsy patients transitioned from child to adult care between tertiary centers compared to patients transferred from the community. Patients aged from 18 to 25 years were divided into 2 groups: Group 1 comprised 170 patients referred from the pediatric tertiary center; and Group 2 had 132 patients referred from the 